\title{
Revista Colombiana de

\section{¿Es importante la cuantificación del flujo de las colaterales sistémico-pulmonares en las cardiopatías congénitas?}

\section{How important is the quantification of systemic-to-pulmonary artery collateral flow in congenital heart disease?}

\author{
Walter Mosquera ${ }^{\mathrm{a}, *}$ y Diana M. Dávalos ${ }^{\mathrm{b}, \mathrm{c}}$ \\ a Cardiología, Pediatría, Hemodinamia, Fundación Valle del Lili, Cali, Colombia \\ b Centro de Investigaciones Clínicas, Fundación Valle del Lili, Cali, Colombia \\ ' Salud pública, Facultad de Ciencias de la Salud, Universidad ICESI, Cali, Colombia
}

Recibido el 7 de agosto de 2014; aceptado el 13 de agosto de 2014

Disponible en Internet el 26 de septiembre de 2014

\begin{abstract}
Los vasos colaterales sistémico-pulmonares son conexiones vasculares anormales entre las arterias sistémicas y el lecho vascular pulmonar, que surgen como forma alternativa de suministro de sangre ante la disminución del flujo sanguíneo pulmonar. Se dan en pacientes con enfermedades congénitas y adquiridas, y se sospechan en recién nacidos prematuros con enfermedad broncopulmonar, pacientes prematuros que hayan requerido cierre del ducto arterioso persistente (farmacológico o quirúrgico), bronquiectasias y enfermedad inflamatoria crónica.

Varían en tamaño, curso, cantidad y distribución, y se originan desde las ramas braquiocefálicas, la pared torácica y la aorta descendente. Algunos mecanismos que contribuyen a su aparición son la hipoxemia, el flujo sanguíneo pulmonar global o regional disminuido y el flujo no pulsátil. Estos mecanismos se observan en formas graves de tetralogía de Fallot, ventrículo único funcional, atresia pulmonar y broncodisplasia pulmonar. En casos específicos como el ventrículo único funcional, su importancia clínica y su óptima
\end{abstract}

Véase contenido relacionado en DOI: http://dx.doi.org/10. 1016/j.rccar.2013.05.001

* Autor para correspondencia.

Correo electrónico: cardio95@hotmail.com (W. Mosquera). evaluación han sido tema de debate durante las dos últimas décadas ${ }^{1}$.

La fisiología del flujo de los vasos colaterales sistémicopulmonares implica efectos tanto benéficos como perjudiciales; puede aumentar el flujo sanguíneo a los segmentos pulmonares hipoperfundidos, mejorar el intercambio gaseoso e inhibir potencialmente el desarrollo de las malformaciones arteriovenosas pulmonares en quienes el flujo venoso hepático no alcanza la circulación pulmonar; sin embargo, también puede generar dilatación ventricular, disfunción y regurgitación de la válvula auriculoventricular, o generar síntomas de falla cardiaca al llevar un flujo mayor pero ineficiente a un ventrículo con circulación univentricular. El exceso de flujo de los vasos colaterales sistémico-pulmonares en el paciente con corazón univentricular se traduce en distrés respiratorio, disfunción ventricular, presión vascular pulmonar alta, gasto cardiaco bajo, hipertensión venosa central, derrames pleurales, ascitis y enteropatía perdedora de proteínas ${ }^{2}$. Adicionalmente, el flujo de los vasos colaterales sistémico-pulmonares puede volver a las venas pulmonares y revelarse en el campo quirúrgico durante el bypass cardiopulmonar.

De otra parte, existen diferencias en el manejo de los vasos colaterales sistémico-pulmonares; en un extremo están aquellos en quienes estos se ocluyen solo en circunstancias muy seleccionadas, generalmente cuando grandes 
colaterales con flujo exuberante se consideran la causa del compromiso clínico del paciente. Por otro lado, están quienes rutinariamente embolizan estas colaterales y describen mejores resultados clínicos, como la corta duración de efusiones pleurales y efusiones pericárdicas postoperatorias, la disminución de la estancia hospitalaria, así como menor disfunción ventricular y mortalidad. Banka et al. ${ }^{3}$ encontraron una incidencia entre 0 y $30 \%$ en el cierre de colaterales sistémico-pulmonares sin observar diferencias significativas en las complicaciones postoperatorias y en la estancia hospitalaria entre los grupos comparados. Hasta hace poco, la dificultad para medir objetivamente y en forma no invasiva el flujo de los vasos colaterales sistémico-pulmonares, generaba un gran debate entre aquellos que rutinariamente realizaban la embolización y quienes no. Los trabajos de Wartmannet et al. reportan la evaluación sistemática de la medición del flujo de los colaterales sistémico-pulmonares por resonancia magnética nuclear, utilizando un mapa de velocidad a través de una planta de contraste de fase ${ }^{4,5}$.

El artículo de Carvajal et al. «Embolización de colaterales en niños con cardiopatías congénitas. Experiencia en un centro cardiovascular» publicado en este número de la revista, describe un grupo de pacientes pediátricos con cardiopatía congénita a quienes se les practicaron embolizaciones de colaterales por vía percutánea. El reporte de las características clínicas, los desenlaces, las complicaciones y los diferentes dispositivos utilizados por este grupo en la embolización de los colaterales permite valorar los resultados de esta técnica de acuerdo con su experiencia de 8 años. Al conocer la información de estos 27 pacientes en quienes se hizo embolización percutánea de 33 colaterales, es posible comparar los desenlaces clínicos de nuestros propios pacientes y tener evidencia de lo que llevan a cabo otros grupos de Cardiología pediátrica en el país.
Al igual que otras experiencias reportadas en la literatura, en estos pacientes con cardiopatías congénitas la embolización de colaterales por vía percutánea es un procedimiento seguro y con alta tasa de eficacia, sin mayor variación de los resultados de acuerdo con los dispositivos utilizados. No obstante, la búsqueda de evidencia que permita definir la importancia de la embolización de colaterales en grupos de pacientes específicos, aún es escasa. Futuros trabajos de investigación enfocados a definir la magnitud del flujo aportado por estas colaterales, brindarán información valiosa para guiar el manejo precoz de estos pacientes e impactar de manera favorable los resultados.

\section{Bibliografía}

1. Inuzuka R, Aotsuka H, Nakajima H, Yamazawa H, Sugamoto $K$, Tatebe S, et al. Quantification of colateral aortopulmonary flow in patients subsequent to construction of bidirectional cavopulmonary shunts. Cardiol Young. 2008;18:485-93.

2. McElhinney DB, Reddy VM, Tworetzky W, Petrossian E, Hanley FL, Moore P. Incidence and implications of systemic to pulmonary collaterals after bidirectional cavopulmonary anastomosis. Ann Thorac Surg. 2000;69:1222.

3. Banka P, Sleeper LA, Atz AM, Cowley CG, Gallagher D, Gilispie MJ, et al. Variability and outcomes of coil embolization of aortopulmonary collaterals before Fontan completion: a report from the Pediatric Heart Network Fontan Cross- Sectional Study. Am Heart J. 2001;162:125-30.

4. Powell AJ, Maier SE, Chung T, Geva T. Phase velocity cine magnetic resonance imaging measurement of pulsatile blood flow in children and young adults: in vitro and in vivo validation. Pediatr Cardiol. 2000:21.

5. Whitehead Sundareswaran KS, Parks WJ, Harris MA, Yoganathan AP. Foge IMA. Blood flow distribution in a large series of Fontan patients: a cardiac magnetic resonance velocity mapping study. J Thorac Cardiovasc Surg. 2009;138(96):102-10. 